\title{
DATA FOR PROBING THE SUN
}

\author{
YVONNE ELSWORTH \\ School of Physics \& Astronomy, University of Birmingham \\ Edgbaston, Birmingham B15 2TT UK \\ E-mail: ype@star.sr.bham.ac.uk
}

\section{Introduction}

The observations of solar oscillations provide an unrivalled, precise way of probing the solar interior. In this paper, I consider the observations and their interpretation in terms of the physics of the Sun. The oscillations that we are concerned with here are the so-called $p$ modes, i.e. oscillations for which pressure is the restoring force. The modes for which gravity is the restoring force have yet to be unambiguously detected on the Sun. The observations are made either as Doppler velocity or as intensity and are, in general, very small effects. To get an impression of the precision required, consider that in integrated velocity the total signal is $\sim 1 \mathrm{~m} \mathrm{~s}^{-1}$ with the strongest individual modes being about $15-20 \mathrm{~cm} \mathrm{~s}^{-1}$. The weakest, detected modes are of order a few $\mathrm{mm} \mathrm{s}^{-1}$. When this signal is measured as a Doppler shift, $v / c$ is a few parts in $10^{11}$. The observations are made by a variety of instruments on Earth or in Space which can be simply divided into those which observe the Sun as a star and those which image the solar surface into many pixels Although there are many different observers using many different techniques, in all cases one is analysing light emitted from a region relatively high in the atmosphere of the Sun. When one considers how these measurements can be interpreted in terms of the solar oscillations, two issues arise:

1. Roughly where in the solar atmosphere are the lines formed?

2. How different are the heights of formation for different lines?

There are three lines which are widely used. They are lines of sodium (IRIS and GOLF), potassium (BiSON and LOWL) and nickel (GONG and MDI) and we will now consider their formation height in the solar atmosphere. The reference point against which we will specify the height at which the lines are formed is given as the height where the local temperature equals the effective temperature of the Sun. This closely corresponds to optical depth equals unity. The temperature minimum is at about $515 \mathrm{~km}$ above this level.

As indicated in Table 1, the nickel and the potassium signals come from very much the same height whereas the sodium signal is formed significantly higher in the atmosphere. To see an implication of the height differences, we need to consider the sound waves themselves. The Sun acts as a resonant cavity for sound waves. The cavity 
TABLE 1. Formation height for several common observing lines

\begin{tabular}{ccc}
\hline Element & Height above zero $(\mathrm{km})$ & Wavelength $(\AA)$ \\
\hline Sodium D1\&D2 & 500 & 5,893 \\
Potassium D1 & 300 & 7,699 \\
Nickel I & 300 & 6,768 \\
\hline
\end{tabular}

is limited by upper and lower turning points which are a function of the particular mode being observed. The upper turning point occurs when the vertical scale of the waves becomes comparable with the scale on which the vertical stratification is changing. This occurs where the frequency of the wave is approximately equal to the acoustic cut-off frequency. The acoustic cut-off frequency increases steadily from the deep interior, where it is small, towards the surface. Hence the upper turning point is located deeper for a low-frequency mode than for a high-frequency mode. For example, a mode whose frequency is $1 \mathrm{mHz}$ would have an upper turning point at $0.987 R_{\odot}$ and one at $4 \mathrm{mHz}$ would turn closer to the surface at $0.999 R_{\odot}\left(R_{\odot}\right.$ is the photospheric radius where the temperature equals the effective temperature.)

At very high degree, the upper turning point is a function of both degree and frequency, and the the simple numbers quoted above cannot be used.

As the excitation of the modes is believed to occur in the very upper layers of the Sun (within a few hundred $\mathrm{km}$ of the surface), the observed widths and strengths of a particular mode of oscillation will depend strongly on the location of the upper turning point. Comparison of the numbers in Table 1 with the turning points shows that, in most cases, the region from which the signal comes is actually outside the sound-wave cavity and we are detecting an evanescent wave. This has consequences for the observed strength of the signal. The density of the solar atmosphere drops with increasing height and the oscillation signal is hence stronger if the line is formed higher up.

So, we expect that oscillations measured with the sodium line are stronger than for those measured with potassium. The relative strengths of the two signals have been measured by Fossat (private communication) to be a function of frequency changing from no difference at all at about $1.5 \mathrm{mHz}$ to a factor of about 1.5 in power at $4.5 \mathrm{mHz}$. We should thus expect that the raw spectra will look different if measured in the different lines.

The lower turning points vary strongly for the different modes considered. The lowest $\ell$ go into the core and the highest $\ell$ are confined to the surface. According to asymptotic theory the inner turning point varies as $\nu / L$ (where $L=\sqrt{\ell(\ell+1)}$ ). One mode which has its inner turning point at the base of the convection zone is $3 \mathrm{mHz}$ $\ell=50$ (Gough, 1990). At lower frequency and the same $\ell$-value the modes are totally confined within the convection zone. On the other hand, at lower degree, a mode with $\ell=1$ and $\nu=3 \mathrm{mHz}$ will get within $0.06 R_{\odot}$ of the centre. Only $\ell=0$ modes probe the very centre of the core. Thus it is an extremely powerful attribute of solar oscillations that the volume of the sun sensed by different modes varies enormously.

However, consideration of the inner turning point of a mode does not tell the whole story for the importance of modes in determining the structure of the Sun. 


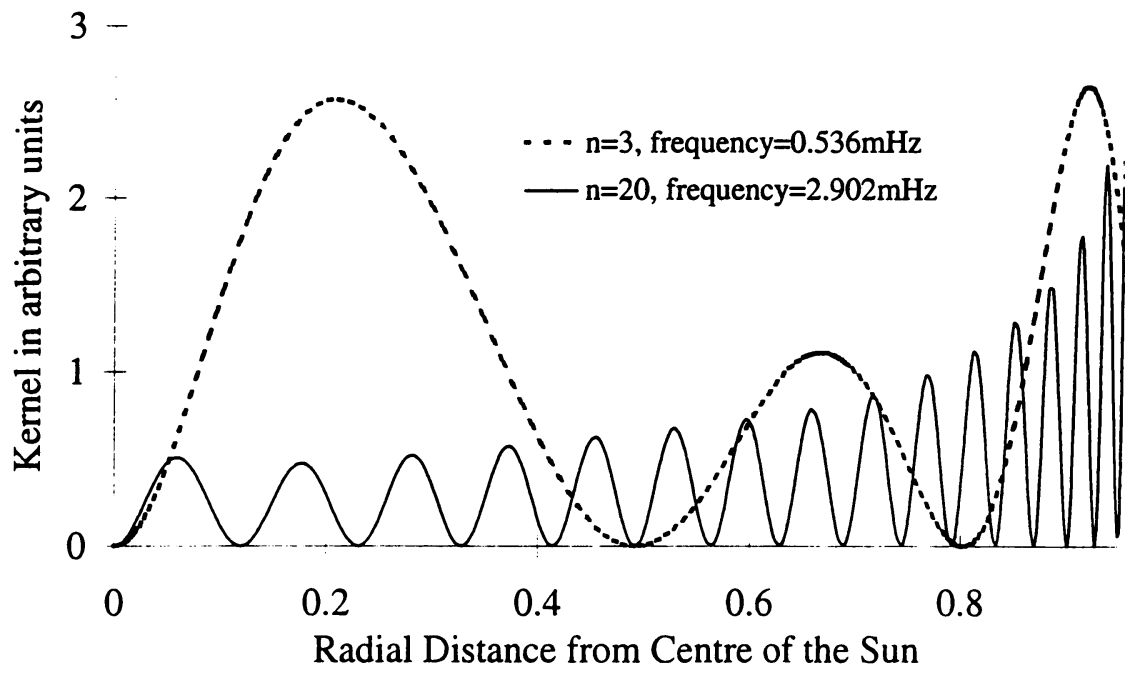

Figure 1. Typical kernels for $c^{2}$ for low-order, zero-degree modes. Note their differing sensitivity in the core

Asymptotic theory predicts that the higher the frequency of a mode of given $\ell$, the closer to the centre of the Sun will it turn, but, one must also consider where the energy of a mode is principally concentrated. Figure 1 illustrates that although the mode of higher frequency does have an inner turning point which is closer to the solar core, the lower frequency mode has much more of its energy in the inner regions of the Sun. These considerations indicate that low-order modes of low frequency are very effective at sensing the conditions in the solar core. One must also take into account the fact that low-frequency-mode peaks are much narrower than high frequency ones and so can be measured much more accurately. Detecting modes of low degree and low frequency remains one of the very important goals of helioseismology.

\section{Seismic Sun}

The oscillation frequencies are sensitive to the conditions inside the Sun. What the oscillations are really measuring the speed of sound as a function of the mode which can be interpreted as a function of radius and latitude in the Sun. The sound transit time is $\int d r / c$ from the lower to the upper turning points, where $c$ is the speed of sound. The time that a mode spends in a particular volume, and hence its sensitivity to the physical conditions there, depends inversely on the speed of sound. Originally this led to the suggestion that $p$-mode oscillations could not be used to sense the core. However the very high precision of the measured frequencies (up to 1 in $10^{5}$ near the centre of the spectrum ) means that we can, in fact, say quite a lot about the core. G-modes would be still better but they have yet to be detected. A picture of the Sun built up from helioseismic data may be termed the seismic Sun. Helioseismology gives direct constraints only on the mechanical properties of the Sun (i.e. determined by pressure, density and gamma). If the equation of state is assumed, or where the solar plasma is fully ionised, the sound speed constraint gives a constraint on the 


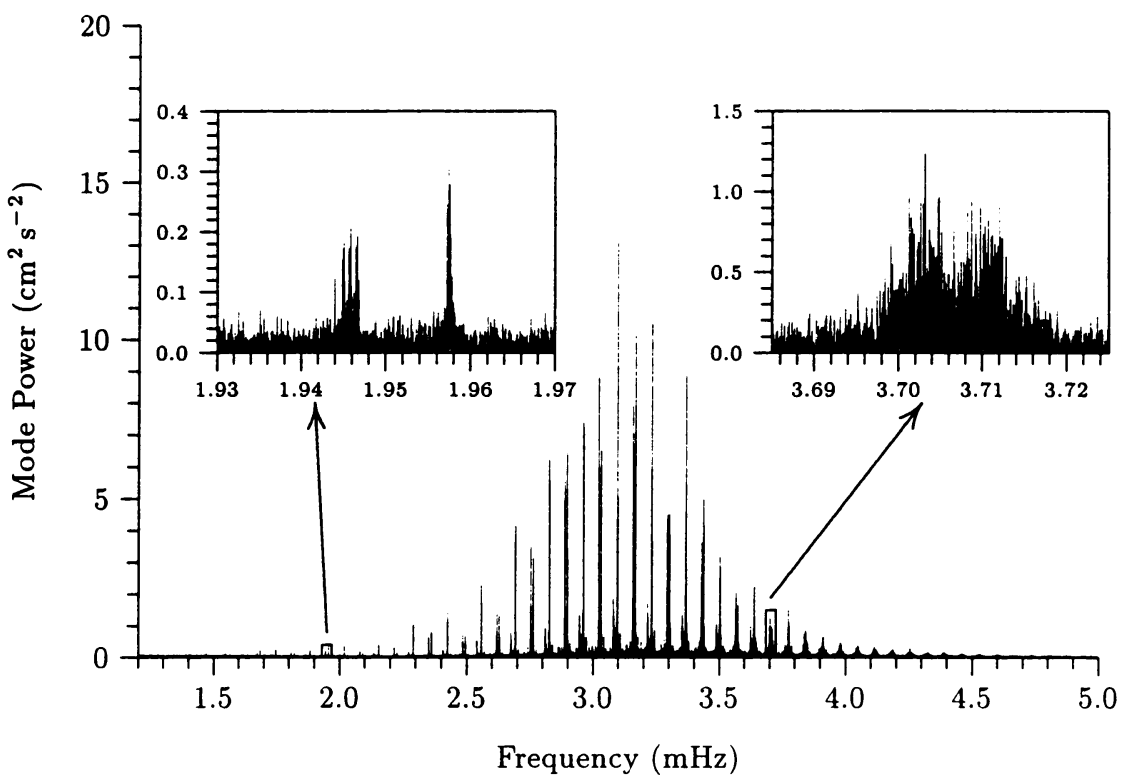

Figure 2. Sixty-four-month BiSON power spectrum.

ratio of temperature to mean molecular weight. To be able to extract information on the thermal structure or composition additional input such as the equation of state, nuclear reaction rates and opacities are required. An important additional point is that a seismic model has the advantage that it looks at how the Sun is now, and does not require one to be able to accurately follow the solar evolution.

\section{Errors in theory and in practice}

So given that the possibility that the frequencies can be used to extract direct information concerning the interior of the Sun, we should explore what limits the accuracy of the frequencies.

A spectrum taken from 64 months of data from the 6-station world-wide BiSON (Chaplin et al., 1996) network is shown in Figure 2. It illustrates the typical range of power and frequency of the low-degree solar oscillations. The 64-month BiSON spectrum shows lines that can be measured from about $1.1 \mathrm{mHz}$ to $4.7 \mathrm{mHz}$.

The spectrum can be divided into three regions: the central region where the signal levels are high; the low frequency end; and the high frequency end. Each region presents its own problems. In the middle of the spectrum, where the signal-to-noise is high, the potential precision is high but great care must be taken with the model used to fit the data (Toutain \& Appourchaux, 1994). At very high frequencies and high $\ell$ there are problems in isolating the modes because the widths of the individual components increase substantially. There is also the issue of overlapping spatial aliases in resolved-Sun data. The key issue at very low frequencies is the signal to noise. If the mode is essentially unresolved by the observation, all the oscillatory power is put into the same frequency bin. Under this condition the time required to detect the mode above the background (say a signal to noise level of three) depends on the 
relative strengths of the background and signal. Once the mode is stronger than the background, the signal to noise ratio will increase linearly with observing time. If the data set is longer than a lifetime and the mode is spread out over more than one bin in the frequency spectrum, then the signal-to-noise improves only very slowly as more data are collected. To detect the mode at all, a good general rule is that one must detect a mode in one life time. It is fortunate that the low-frequency modes also have long lifetimes and so long integration times (many months) can be used.

Ground-based data nearly always have gaps. Breaks in the data in the first instance give rise to sidebands at a frequency that is characteristic of the typical interval - daily breaks lead to structure at $11.57 \mu \mathrm{Hz}$. But breaks in the data also give rise to general noise. For nearly $100 \%$ data coverage one has to turn to the data measured on the SOHO satellite. The GOLF 8-month spectrum (Lazrek et al., 1997) has noise levels of order $50 \mathrm{~m}^{2} \mathrm{~s}^{-2} \mathrm{~Hz}^{-1}$ at $1 \mathrm{mHz}$. In their early spectra, taken before the failure of the rotator, the noise level is about a factor of two lower. Direct comparison with BiSON is a little difficult because the data have been taken at different wavelengths but at these frequencies direct comparison should be possible. BiSON solar noise (Elsworth et al., 1994) estimates from cross correlations of short data sets, and also observed levels in a one-day spectrum on a good day at their best site, indicate that the noise level is at about $20 \mathrm{~m}^{2} \mathrm{~s}^{-2} \mathrm{~Hz}^{-1}$ at $1 \mathrm{mHz}$. Their 32 -month spectrum has a power level of $70 \mathrm{~m}^{2} \mathrm{~s}^{-2} \mathrm{~Hz}^{-1}$ at $1 \mathrm{mHz}$. Some of the difference between the two estimates of the noise is due to the effects of the atmosphere but the lack of a $100 \%$ fill is also relevant. Simulations suggest that the noise level at $1 \mathrm{mHz}$ will rise by a factor of 10 when the fill changes from $100 \%$ to $74 \%$ (Chaplin et al., 1997). In reality, tests on real data suggest that the presence of solar noise may limit the degradation. In so far as these simulations are applicable, they suggest that the gaps are a significant source of noise in a computed spectrum. Typical fills achieved by the BiSON observations are of order $80 \%$ and the GONG observations are more like $90 \%$ over long periods. Given the indications from simulations, gap filling should be a very profitable procedure. Many of the gaps in network data are quite short and so should be amenable to data-filling techniques (Brown \& Christensen-Dalsgaard, 1990).

The normal fitting procedure is to fit to a Lorentzian line shape with either $\chi^{2} 2$ d.o.f. or gaussian statistics as appropriate. Using the correct statistics has been shown to be very important (Toutain \& Appourchaux, 1994). Early indications that the lines were not well described by a symmetric function came from the high- $\ell$ data and this is now extended to a wide range of modes. It is now clear that at least some of the systematic differences between different data sets are dependent on the shape of the lines. The line-shape effects are different between intensity and velocity, giving rise to frequency discrepancies of low-degree modes as measured in intensity and velocity of about $0.1 \mu \mathrm{Hz}$ with intensity giving higher frequencies than velocity. This is discussed elsewhere (Toutain et al., 1997; Appourchaux et al., 1997). Solar noise (see Figure 3) is the ultimate background against which the measurements must be made (Elsworth et al., 1994; Underhill \& Isaak, 1997; Fröhlich et al., 1997). However, in reality one must consider also other sources of noise such as instrumental, photon shot noise, and atmospheric noise. The relative importance of these various noise sources depends on the region of the spectrum and the type of observation.

Theoretical estimates of the errors can be made. A simple derivation of the expected frequency error is given elsewhere (Elsworth et al., 1994) as is a more precise derivation which takes account of the signal to noise in the data (Libbrecht, 1992). Simply, assume that a mode is detected in one life-time. A crude estimate of the error on this measurement is one half of the line width. One then continues to observe and 


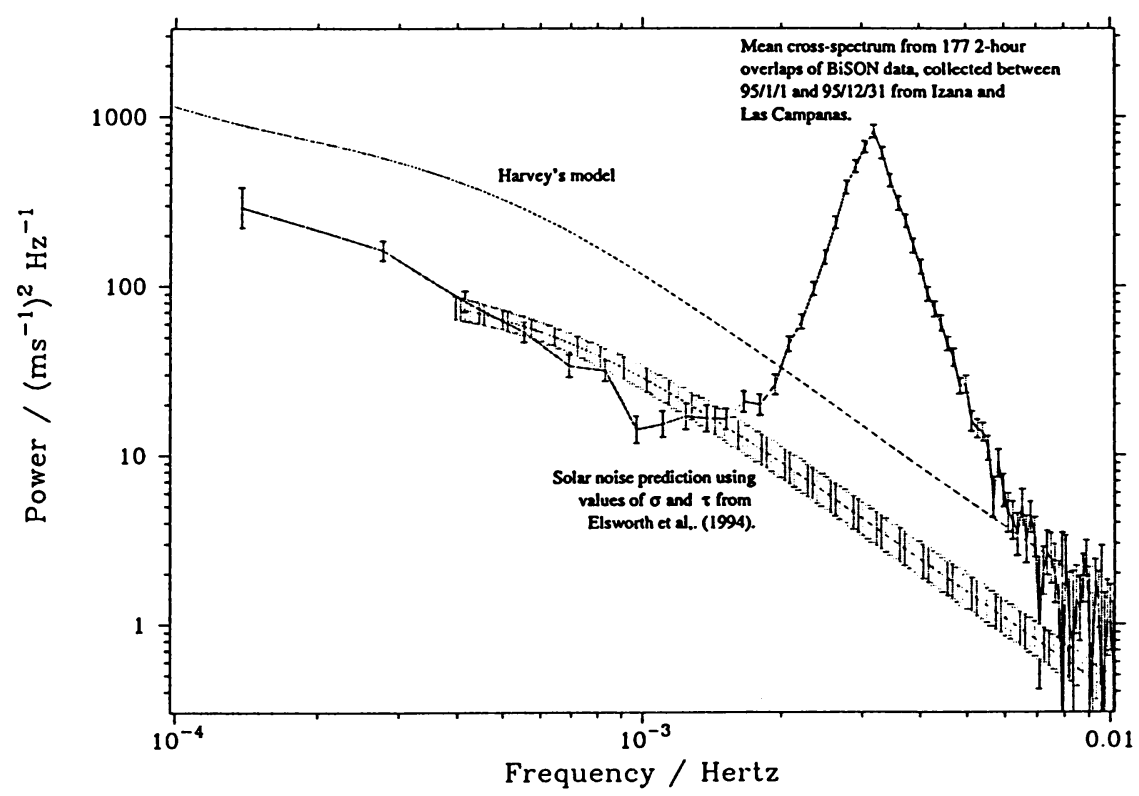

Figure 3. Solar noise spectrum as measured by BiSON using cross-correlation techniques

amasses data for $\mathrm{n}$ lifetimes. The data are adding incoherently and so the uncertainty in the frequency can be expected to have improved by $\sqrt{n}$. Putting this together we find that the error $=\sqrt{(\Gamma / 4 \pi t)}$ where $\Gamma$ is the full width at half maximum of the line and $t$ is the total observing time. This is the best that the error can be expected to be: poor signal to noise increases the error.

There are several points to be made here. First, if the signal to noise is good then the error on the frequency estimation is a slow function of improving signalto-noise. Second, the error will scale with the square root of the line width which is itself a strong function of frequency. We can check how close the real data get to this prediction by looking at some existing datasets.

Figure 4 shows the line width and frequency errors as a function of frequency for BiSON, GOLF and MDI for $\ell=0$.

On the same graph are shown the predictions of the formula above. The BiSON spectrum is taken over 32 months and there is a prediction for a dataset of this duration. The GOLF spectrum is taken over 8 months and the MDI over 12 months. In all cases, the observations are close to the predictions in the centre part of the spectrum where the signal to noise is good. At the extremes of the frequency range there are still improvements to be made. In the case that the error in the frequency determination is greater than the linewidth, we can say that there is probably not a secure detection of the mode. Where the error is close to the theoretical prediction, improving the signal-to-noise in the data will only have a marginal effect on the frequency accurary. The main improvement will come from having much longer data series. This is illustrated by the comparison between the BiSON errors and those from the other two, shorter spectra. The scatter in the measurements may indicate 


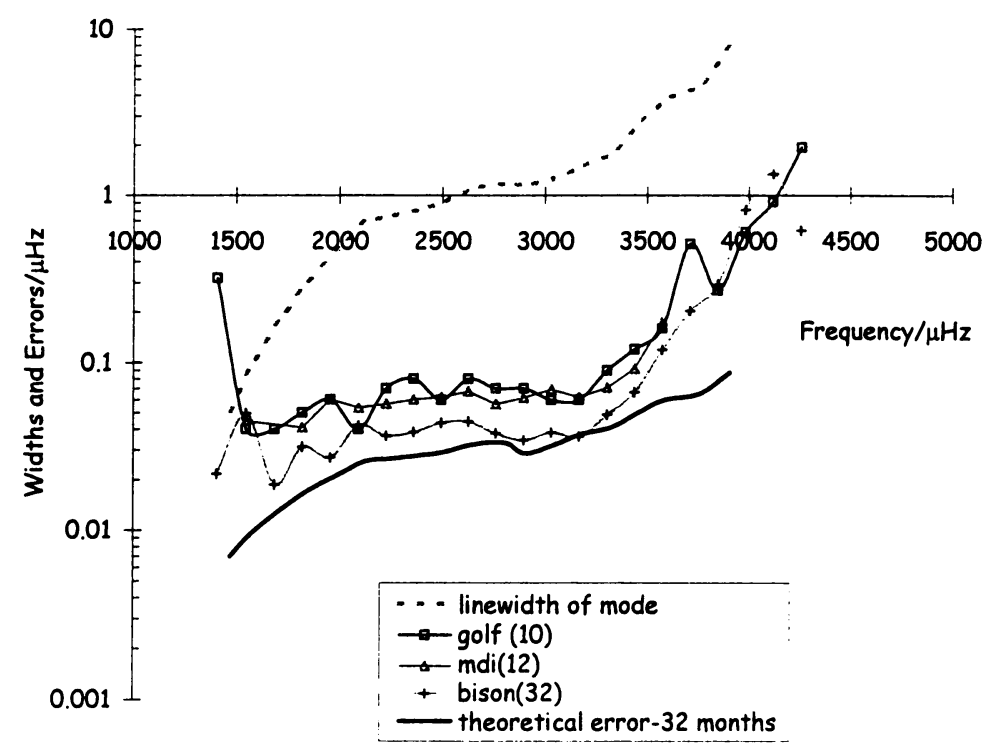

Figure 4. Frequency errors for $\ell=0$, for different data sets

that the constraints used in the fitting are not quite right. In specifying the error on a parameter in reality, one has to consider internal and external (i.e. taking into account the data scatter) errors.

In this discussion we have not yet considered frequency changes due to solar magnetic effects in the short and long term. The solar cycle has been shown to cause the mode frequencies to vary with time (Libbrecht \& Woodard, 1990; Elsworth et al., 1990). Currently, we are near the bottom of a solar cycle and hence recent observations have not been much influenced by the effects of solar activity. An important point for the consideration of errors is how long a data set can be considered before the variation in the activity levels on the Sun must be considered. As far as the frequencies are considered, we specify that the bin width of the fourier transform should be greater than any frequency shift. At the bottom of the solar cycle, this constraint still allows us to use a 32 -month spectrum.

\section{Standard Model and its Physics}

When setting up a standard solar model, the primary requirement is to make the model look like the real Sun. By this we mean that we must match the mass, radius and luminosity of the current real Sun. We also put in the physics that we believe to properly describe the behaviour of the solar interior. We do not perfectly understand the interior of the Sun and so there are areas for further development of the theory. In the energy-generating core there is the problem that the observed neutrino rates do not match the predicted rates. In the convection zone there is the general lack of a good analytical description. In most models, mixing length theory is used. There have been improvements recently in the treatment of the superadiabatic layer and turbulent convection. Another parameter that has to be supplied is the opacity as 
a function of radius. There have been significant changes recently in our knowledge of the opacity (Seaton et al., 1994; Rogers \& Iglesias, 1992). The high precision of the solar oscillations frequencies required an improvement to the opacities so that the data would more closely match the theoretical predictions. A spin off has been that the changes made have allowed an improvement to the modelling of other stars and have removed certain long-standing problems. Low temperature opacities required in the outer layers of the Sun remain a problem.

One of the early successes of helioseismology was that it allowed an accurate determination of the depth of convection zone. Changing other physics will often require the depth of the zone to be changed. However, it has been shown (Basu \& Antia, 1995) that a change in the convection zone by any means is not enough to improve the results. The base of the convection zone is believed to be at $0.713 \pm$ $0.001 R_{\odot}$ if one does not consider the effects of diffusion of helium and other heavy elements. This changes to about $0.711 R_{\odot}$ for model S (Christensen-Dalsgaard et al., 1996) when the effects of diffusion are included.

The equation of state determines the relationship between $P, \rho$ and $\%$. The solar plasma is an almost ideal gas with gamma, the adiabatic exponent, equal to $5 / 3$ everywhere except in the ionisation zones. Away from this condition. we need to know gamma. Recently, the opal project (Rogers \& Iglesias, 1992a) has produced a new way of calculating the adiabatic exponent. This is somewhat of an improvement on the previous method (Mihalas et al., 1988). However there is still considerable discussion of the two methods and particularly how one generates information which is valid and accurate enough to cope with a range of stellar conditions.

Spectroscopic measurements of the abundance of helium in the Sun are very uncertain because the absorption lines are not in the visible region of the spectrum. Observing from above the atmosphere at short wavelengths gives the coronal levels which then have to be linked to photospheric levels. Again this is not an easy problem. The helium abundance can be obtained helioseismically from the variation in the adiabatic index of the solar material in the second helium ionisation zone (at depth of $15,000 \mathrm{~km}$ ). The results from a variety of methods do not agree (Vorontov et al., 1991; Kosovichev et al., 1992; Antia \& Basu, 1994) but are in the range 0.24 to 0.25 helium by weight. This is compatible with solar evolution theories only if helium settles out of the envelope into the radiative zone (Noerdlinger, 1977; Cox et al., 1989; Wambsganss, 1988; Christensen-Dalsgaard et al., 1993). In the absence of settling the present day helium abundance in the solar envelope would have to be about 0.27 to 0.28 to satisfy the solar luminosity constraints.

\section{Comparison with observation}

The physics in the standard model can be used, with ideas of stellar evolution, to get a picture of the Sun for which oscillation frequencies can be computed. These can be compared with the real observed frequencies.

The agreement looks quite good at first sight if the predicted frequencies are marked on a spectrum. However, the data can plotted in a more sensitive way on a so-called echelle plot. In an echelle plot, the spectrum is divided up into slices of a fixed length which are then stacked above each other. If the interval is chosen to be about $135 \mu \mathrm{Hz}$ then the different radial orders are approximately above each other on the plot. The 32-month BiSON spectrum shown is compared with recent frequencies calculated by Guzik and Swenson (1997)in Figure 5. Note that the agreement is 


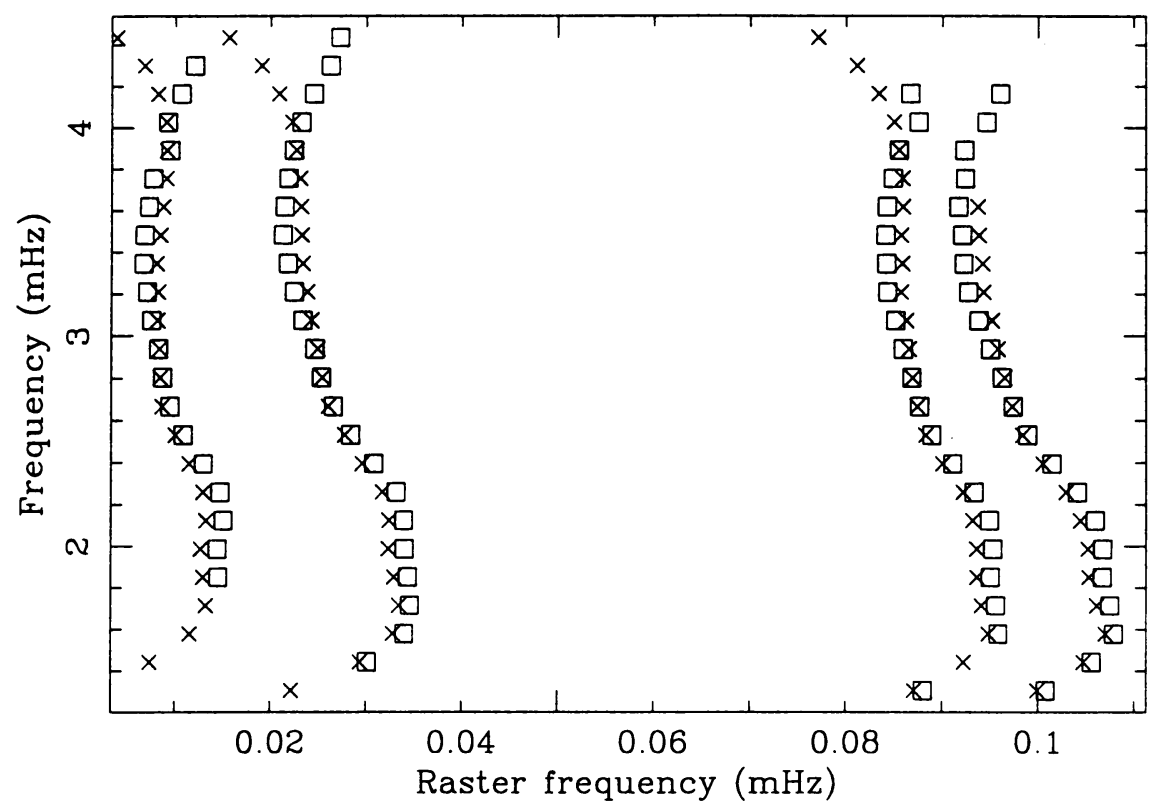

Figure 5. Echelle plot of a 32-month BiSON spectrum and theoretical frequencies from Guzik and Swenson. The squares denote frequencies measured by BiSON from 1994 May 16 to 1997 January 10. The crosses denote model frequencies (Guzik \& Swenson, 1997).

good in the region about $3 \mathrm{mHz}$ and deteriorates somewhat at lower frequencies and more seriously at at higher frequencies. At high frequencies, the lack of agreement is probably due to the difficulties in modelling the solar surface. Other models give even better low-frequency agreement. However, one of the great advantages of the Guzik and Swenson code is that it is analytic and can be easily extended to other stars.

Similarly with the high-degree data, the agreement between theory and experiment is a function of frequency. When comparing modes of very different $\ell$ it is useful to scale them by the mode inertia (Christensen-Dalsgaard \& Berthomieu, 1991) which takes account of the volume of the Sun sensed by the mode and the ease with which the mode can be perturbed. Figure 6 (Christensen-Dalsgaard et al., 1996) is taken from the issue of Science in which many early GONG results were reported. It shows frequency differences between the Sun as measured by the GONG project and a model (model C of JCD). The differences are scaled by $Q_{n l}$ (the mode inertia). As is the case for the low-order data, the discrepancies between the real Sun and the model are most marked at higher frequencies. There is some residual $\ell$-dependence which can be used to localise the region in which the physical description of the Sun is not accurate. In this particular case, there appears to be a problem at the base of the convection zone.

Small differences between data sets need to be understood and we need to know how to extract 'true' frequencies from asymmetric lines. There is also much more work to be done on solar models. However, one can say in conclusion that the disagreement between theory and observation, although at the few per cent level, is larger than the errors on the data and is hence very significant. 


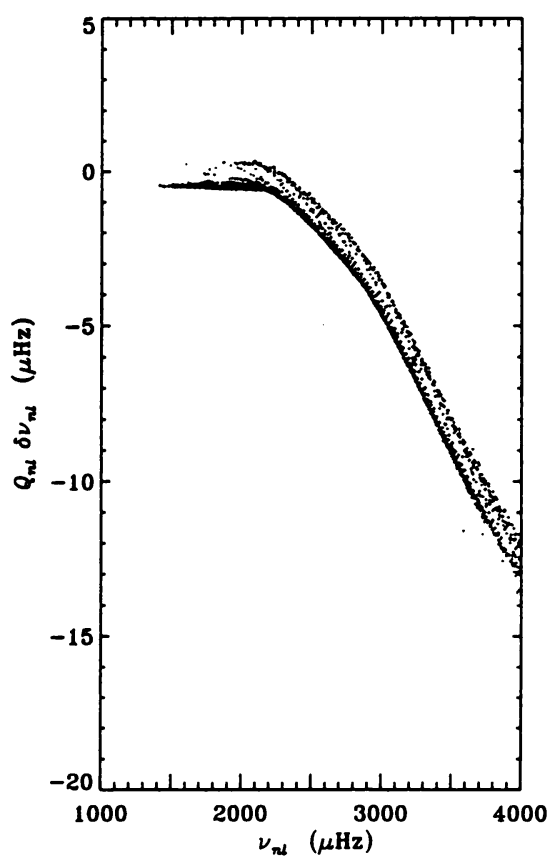

Figure 6. GONG frequencies scaled by mode inertia compared with model $\mathrm{C}$

\section{The Core}

As has been apparent for over a decade, there is a problem with the rate at which the neutrinos from the Sun are observed at the Earth. At first it was felt that the problem was with our understanding of the solar physics of the core. However, the observed spacing between adjacent modes $(\ell=0$ and $\ell=2$ for example) are very close to the predicted spacing. All attempts to modify the theoretical separation in a way which will lower the expected neutrino flux have produced frequency separations which are excluded at high significance by the data. The importance of using the low- $\ell$ frequency differences is two fold. First the modes probe deep into the Sun with the $\ell=0$ mode being deeper than the $\ell=2$. Secondly, the only significant differences in the volumes sensed are in the interior of the Sun. The surface effects, which are very hard to model, have very little impact on the frequency differences (Elsworth et al., 1990). These same frequency differences can be used to explore the effects of gravitational settling of Helium and heavy elements. The data clearly support their incorporation.

The small and large frequency separations can be parameterized in a way which allows comparison of large and small separations to determine stellar ages since nuclear burning makes the core conditions very sensitive to the evolutionary state of the star (Christensen-Dalsgaard, 1984). 


\section{Solar structure inversions}

Another approach to the problem is that of inversions. The data can be inverted to give the sound speed profile through the Sun. Other papers in this volume discuss the technique in much more detail. The inversions use not only the frequencies but also the errors on the frequencies to determine the solar parameters. An observational error which is unrealistically small will bias an inversion as will errors which are too large. It is crucially important to have realistic error estimates. Differential inversions address the problem of the difference in a particular parameter under consideration between the Sun and a model of the Sun whose physics is thought to be an approximation to that in the Sun.

The inversions tell a similar story to the one given above; the solar models match the data well but there are still differences and these are located at the base of the convection zone and in the core of the Sun (Turck-Chièze et al., 1997; Bauu et al., 1997). The sound speed is higher in the Sun than in the reference model just below the base of the convection zone. This could be due to the accumulation of excess helium below the convection zone (Gough et al., 1996). Model S does not include mixing below the base of the convection zone. If there were mixing then the helium abundance locally would be reduced, decreasing the mean molecular weight, increasing the sound speed and thereby bringing the Sun and the model into better agreement. There are other ways of achieving this, for instance, selective changes in the opacity (Tripathy et al., 1997). Changing the opacity alters the depth of the convection zone which has to be adjusted to meet the constraint that the modelled Sun must look like the real Sun. In the core, the inversion errors get quite large because the number of modes that actually penetrate into the region is rather small. The result here is quite sensitive to the particular mode set chosen. Only the lowest-degree modes sample this region and even those sample the core for a very short time because the sound speed is relatively high in the core. The results in the core are also very sensitive to the low frequency data.

\section{Summary}

Although it is attractive to be able to predict frequencies which accurately match those observed, the real goal is to understand the physics of the solar interior. For the Sun, we have access to a huge amount of data. For other stars, we have a much more limited set. Not only does the faintness of other stars make the problem more difficult, but the range of modes observable is much more limited. In trying to understand how we can interpret future asteroseismic data it is constructive to see what information can be extracted from the Sun using similar data sets to those we can expect to have for stars.

\section{References}

Antia H. M. \& Basu S., 1994, ApJ, 426, 801.

Appourchaux T., Chaplin, W. J., Elsworth, Y., Isaak, G. R., McLeod, C. P., Miller, B. A. \& New, R., 1997, these proceedings.

Basu S. \& Antia H. M., 1995, MNRAS, 276, 1402.

Basu S., Chaplin W. J., Christensen-Dalsgaard J., Elsworth Y., Isaak G. R., New R., Schou J., Thompson M. J. \& Tomczyk S., 1997, MNRAS, in press.

Brown T. M. \& Christensen-Dalsgaard J., 1990, ApJ, 349, 667. 
Chaplin W. J., Elsworth Y., Isaak G. R., McLeod C. P., Miller B. A. \& New R., 1996, Sol. Phy., 168, 1.

Chaplin W. J., Elsworth Y., Isaak G. R., McLeod C. P., Miller B. A. \& New R., 1997, these proceedings.

Christensen-Dalsgaard J., Proffitt C. R. \& Thompson M. J., 1993, ApJ, 403, L75.

Christensen-Dalsgaard J. \& Berthomieu G., 1991, in: Solar Interior and Atmosphere, eds. Cox A. N., Livingston W. C., Matthews M., Univ. of Arizona Press, Tuscon, p. 401.

Christensen-Dalsgaard J., 1984, in: Space Research Prospects in Stellar Activity and Variability, ed. Praderie F., Paris Observatory Press, p. 11.

Christensen-Dalsgaard J. et al, 1996, Science, 272, 1286.

Cox A. N., Guzik J. A. \& Kidman R. B., 1989, ApJ, 342, 1187.

Elsworth Y., Howe R., Isaak G. R., McLeod C. P. \& New R., 1990, Nature, 347, 536.

Elsworth Y., Howe R., Isaak G. R., McLeod C. P., \& New R., 1990, Nature, 345, 322.

Elsworth Y., Howe R., Isaak G.R., McLeod C. P., Miller B. A., New R., Speake C. C. \& Wheeler S.J., 1994, MNRAS, 529, 537.

Elsworth Y., Howe R., Isaak G. R., McLeod C. P., Miller B. A., New R., Speake C. C. \& Wheeler S. J., 1994, ApJ, 434, 801.

Fröhlich C. et al., 1997, Sol. Phys., 170, 1.

Gough D. O., 1990, in: Progress of Seismology of the Sun and Stars, p. 302, eds. Osaki Y. \& Shibahashi H., Springer-Verlag, Berlin.

Gough D. O. et al., 1996, Science, 272, 1281.

Guzik J. A. \& Swenson F. J., 1997, $A p J, \mathbf{4 9 1}$, in press.

Kosovichev A. G. et al., 1992, MNRAS, 259, 536.

Lazrek M. et al., 1997, Sol. Phys., in press.

Libbrecht K. G. \& Woodard M. F., 1990, Nature, 345, 779.

Libbrecht K. G., 1992, ApJ, 336, 1092.

Mihalas D. M., Dappen W., Hummer D. G., 1988, $A p J, \mathbf{3 3 1}, 815$.

Noerdlinger P. D., 1977, $A \mathscr{E} A, \mathbf{5 7}, 407$.

Rogers F. J. \& Iglesias C. A., 1992, $A p J, 401,360$.

Rogers F. J. \& Iglesias C. A., 1992, ApJ Supplement., 79, 507.

Seaton M. J., Yan Y., Mihilas D., Pradhan A. K., 1994, MNRAS, 266, 805.

Toutain T. \& Appourchaux T., 1994, $A \& A, \mathbf{2 8 9}, 649$.

Toutain T., et al., 1997, Sol. Phys., in press.

Tripathy S. C., Basu S. \& Christensen-Dalsgaard J., in: Proc. IAU Symp. 181, Sounding solar and stellar interiors, eds. Schmider F.-X. \& Provost J., Nice Observatory, France, in press.

Turck-Chièze S. et al., 1997, Sol. Phys., 175, in press.

Underhill C. J. \& Isaak G.R., in: Proc. IAU Symp. 181, Sounding solar and stellar interiors, eds. Schmider F.-X. \& Provost J., Nice Observatory, France, in press.

Vorontsov S. V., Baturin V. A., Pamyatnykh A. A., 1991, Nature, 349, 49.

Wambsganss J., 1988, $A \mathscr{E} A, 205,125$. 